\title{
Uma estratégia de proteção eficiente em EONs-SDM utilizando p-cycle FIPP
}

\author{
Helder M. N. da S. Oliveira ${ }^{1}$, Nelson L. S. da Fonseca ${ }^{2}$ \\ ${ }^{1}$ Instituto de Ciências Exatas e Naturais - Universidade Federal do Pará (UFPA) \\ Belém 66075-110, PA, Brasil \\ ${ }^{2}$ Instituto de Computação - Universidade Estadual de Campinas (UNICAMP) \\ Campinas 13083-852, SP, Brasil \\ heldermay@ufpa.br ${ }^{1}$, nfonseca@ic.unicamp.br ${ }^{2}$
}

\begin{abstract}
Emerging applications and services have led to tremendous traffic growth in the core of the Internet and, consequently, there is need to increase the capacity of these networks. Spatial division multiplexing is a promising solution for dealing with the expected capacity depletion of these networks. Additionally, the enormous amount of traffic on these networks requires protection against failures. This paper proposes a routing and protection algorithm for spatial multiplexing elastic optical networks using FIPP p-cycle, minimal interference, and multipath routing. Results indicate that the proposed algorithm produces a better performance when compared to the performance of existing algorithms in the literature.
\end{abstract}

Resumo. O fluxo contínuo de aplicações e serviços digitais emergentes tem impulsionado um enorme crescimento de tráfego no núcleo da Internet e consequentemente o estudo de tecnologias para aumentar a capacidade dessas redes. A multiplexação por divisão espacial é uma solução promissora para lidar com o esgotamento esperado da capacidade dessas redes. Adicionalmente, a enorme quantidade de tráfego nestas redes acarreta na necessidade de proteção contra falhas, uma vez que essas são relativamente frequentes. Neste artigo, propõe-se um algoritmo de roteamento e proteção em redes ópticas elásticas com multiplexação espacial, utilizando p-cycle FIPP, interferência mínima e roteamento multicaminho. Os resultados indicam que o algoritmo proposto produz um melhor desempenho quando comparado ao desempenho dos algoritmos existentes na literatura.

\section{Introdução}

A crescente popularidade de tecnologias como computação em nuvem, vídeo sob demanda, internet das coisas e de outros serviços emergentes da Internet tem levado ao crescimento de tráfego da rede [Forecast 2019]. No entanto, a utilização da capacidade de transmissão da fibra monomodo padrão (SMF) diminuiu drasticamente devido a proximidade cada vez maior do limite não-linear de Shannon das SMFs [Shannon 1948]. Para satisfazer a demanda futura de tráfego, a tecnologia de multiplexação por divisão espacial (SDM) emergiu como uma solução promissora para o problema da limitação de banda da Internet. A tecnologia SDM possui grande flexibilidade na alocação de recursos, maior capacidade e atribuição de espectro. A SDM envolve o uso de canais espaciais e pode ser realizada usando fibra multimodo (MMF), fibra multicore (MCF), ou ainda variações 
destas. No MMF, um única fibra com único núcleo suporta um número de modos que depende do tamanho do núcleo e do índice de refração do revestimento da fibra. Na MCF, uma fibra possui vários núcleos, onde cada núcleo atua como uma fibra de modo único.

Nas redes SMFs, a utilização de enlaces paralelos pode aumentar a capacidade da rede, mas também aumenta o custo do sistema e o consumo de energia. Para satisfazer as demandas de capacidade de comunicação, os equipamentos de transmissão devem reduzir tanto a energia quanto o custo por bit. Comparada com as redes com enlaces paralelos, a integração de componentes é o recurso crucial dos sistemas SDM, pois pode reduzir significativamente o custo e o consumo de energia dessas redes. Nas redes SDM, os componentes integrados no domínio espacial, incluindo transponders, amplificadores ópticos e elementos de rede como comutadores ópticos, podem compartilhar componentes da rede entre canais espaciais para reduzir despesas de capital (CAPEX) e operacionais (OPEX) .

A utilização de MCF com redes ópticas com multiplexação por divisão de comprimento de onda (WDM) foi proposta em meados da década de 80. No entanto, as características de redes WDM não foram consideradas viáveis para utilização conjunta com SDM. As redes WDM empregam alocação de espectro em unidades de tamanho fixo, denominadas de comprimento de onda. O provisionamento de comprimento de onda fixo leva à subutilização do espectro, uma vez que a demanda dos fluxos de dados raramente coincidirão com a largura de banda exata oferecida. As redes WDM permitem que vários fluxos de tráfego compartilhem a banda passante de um caminho óptico, através da agregação de tráfego, no entanto esta técnica não elimina o desperdício de recursos e requer gerenciamento em tempo real de eventuais agregações de tráfego. As redes ópticas elásticas (EONs) distingue-se das redes ópticas WDM pela sua granularidade variável e grade flexível. Em redes WDM, as frequências do canal são fixas (normalmente espaçadas de 25 ou $50 \mathrm{GHz}$ ) e a largura de banda de cada canal é geralmente muito maior que a largura de banda solicitada de um serviço, levando a um desperdício na utilização do espectro. As redes EONs empregam uma grade flexível com frequências centrais espaçadas com 12,5 GHz e multiplexação por divisão de frequência ortogonal (OFDM) para reduzir o nível de alocação de largura de banda.

As EONs convencionais utilizam as dimensões de tempo e frequência para fornecer recursos de redes ópticas com demanda de tráfego variável. A adição de uma terceira dimensão é realizada através do uso de SDM e consequentemente múltiplos núcleos de fibra. A utilização de redes ópticas elásticas com multiplexação por divisão espacial (SDM-EONs) com MCF abre espaço para a comutação de núcleo. Na literatura três abordagens de comutação de núcleo tem sido proposta [Marom et al. 2017]: 1) comutação independente (InS), na qual todos os slots de frequência (FSs) e modos espaciais podem ser, independentemente, dirigido para qualquer porta de saída; 2) comutação conjunta (JoS), na qual todos os modos/núcleos espaciais são tratados como uma única entidade, enquanto os FSs podem ser alternados livremente pelo comutador; e 3) JoS fracionário (FJoS), na qual alguns grupos de modos espaciais e todos os FSs podem ser alternados independentemente para todas as portas de saída.

Um dos problemas fundamentais de EON é o problema de roteamento e alocação de espectro (RSA), que deve considerar a alocação contínua e contígua do espectro para todos os enlaces de um caminho óptico [Pederzolli et al. 2019]. A inclusão de SDM acrescenta outra dimensão ao problema tradicional de RSA, que passa a ser o problema de roteamento e alocação de núcleo e espectro (RSCA). Ao considerar o MCF, a formulação 
de RSCA também deve considerar o crosstalk produzida pela propagação na mesma banda do espectro em núcleos adjacentes. Além disso, a comutação de núcleos pode atenuar a restrição de continuidade do espectro em comparação com o RSA, pois existem vários núcleos que podem ser usados em um enlace de fibra. Em outras palavras, um caminho óptico de uma fonte para seu destino pode ter um espaço de roteamento maior.

Com o estabelecimento e encerramento de conexões em redes EONs, o espectro tende a ficar fragmentado, o que diminui a possibilidade da sua utilização, aumentando, consequentemente, o bloqueio de conexões. A fim de mitigar o impacto da fragmentação do espectro, o roteamento multicaminho foi utilizado em redes EONs. $\mathrm{O}$ roteamento multicaminho consiste em usar mais de um caminho para rotear os bits de um fluxo. O roteamento multicaminho tem sido usado em redes WDM para acomodar demandas de largura de banda acima do comprimento de onda, bem como para fornecer proteção às conexões. Em EON-SDM, o roteamento multicaminho pode tirar proveito da alocação de granularidade fina do espectro, bem como da dimensão do espaço, especialmente quando empregando MCF. Em EONs o roteamento multicaminho reduz o bloqueio de solicitações ao estabelecimento de conexão, pois usa vários caminhos para fornecer a largura de banda necessária quando não é possível atender à demanda com a largura de banda disponível em um único caminho. A utilização de roteamento multicaminhos em EONs oferece vantagens de maior utilização do espectro, menor probabilidade de bloqueio de banda larga [Alvizu et al. 2018], e incremento de sobrevivência [Zhan et al. 2019, Oliveira and da Fonseca 2019a], já que o volume total da demanda está dividido em diversas transmissões por caminhos diferentes.

Uma característica da utilização de roteamento multicaminho é que a divisão do tráfego em múltiplos caminhos gera retardo diferencial, causado pelos diferentes retardos de propagação, o que acarreta a chegada de pacotes fora de ordem no nó de destino, requerendo memória para o re-sequenciamento desses pacotes [Drummond et al. 2017]. Neste artigo, assume-se que os nós destino possuem a quantidade de memória necessária para que as aplicações em tempo real não sejam afetadas. Além disso, consideramos a abordagem MCFs devido às vantagens de geração de menor crosstalk, independência entre núcleos e maior custo benefício. Adicionalmente, visando reduzir o CAPEX, a alocação de recursos considera os multiplexadores ópticos add-drop reconfiguráveis (ROADM) mais simples, que não conseguem utilizar diferentes núcleos para o mesmo caminho, desta forma é necessário manter a contiguidade e continuidade de núcleo e espectro e o formato de comutação JoS.

A capacidade de sobrevivência é uma questão importante para as redes ópticas. Existem diversos métodos de proteção [Assis et al. 2019, Oliveira and da Fonseca 2019b, Gao et al. 2019], sendo a proteção de p-cycle particularmente atraente devido à sua alta velocidade de restauração e eficiência de proteção [Oliveira and da Fonseca 2019b, Xiao et al. 2019]. Os p-cycles fornecem proteção contra falhas usando estruturas em forma de anel pré-conectadas. A velocidade de restauração dos p-cycles deve-se ao fato de os comutadores no caminho de proteção estarem pré-conectados e apenas os nós em que o tráfego é desviado para os caminhos de backup precisarem ser configurados na ocorrência de uma falha. Um tipo de $p$-cycle de particular interesse é o $p$-cycle de proteção de caminho independente de falhas (FIPP). No FIPP, em caso de falha, o tráfego é desviado para um caminho de backup no $p$-cycle no nó de origem. Nesse caso, um $p$-cycle fornece proteção a todos os caminhos de trabalho entre qualquer par de nós que estão no $p$-cycle, 
e o caminho de proteção é o mesmo para um caminho de trabalho, independentemente do enlace no caminho que falhar.

O desempenho dos p-cycles depende de diversos fatores, como o tamanho do ciclo, o número de enlaces no ciclo e a topologia da rede utilizada. A escolha dos enlaces na formação do $p$-cycle FIPP é de grande importância, uma vez que a má escolha dos enlaces pode levar a uma rápida saturação da rede. Para manter uma utilização equilibrada dos recursos de rede na formação do $p$-cycle foi proposta a utilização de interferência mínima. A ideia é gerar $p$-cycles transzonais aos caminhos primários minimizando a taxa de rejeição das requisições futuras. Os $p$-cycles FIPP transzonais são os $p$-cycles que protegem caminhos que têm seus nós finais no ciclo. Diferentemente do algoritmo proposto em [Oliveira and da Fonseca 2019a] que utiliza $p$-cycles FIPP para prover proteção aos caminhos primários, o algoritmo MINION adicionalmente utiliza interferência mínima na criação do $p$-cycle, priorizando $p$-cycles que usam enlaces disjuntos do caminho protegido ( $p$-cycles transzonais).

Neste artigo, propõe-se um algoritmo de proteção para redes ópticas elásticas com multiplexação espacial utilizando roteamento híbrido. Roteamento híbrido é a política de roteamento que primeiro tenta alocar um único caminho para uma requisição e aloca múltiplos caminhos somente se a alocação de um único caminho não for possível. Além disso, o algoritmo proposto utiliza os conceitos de $p$-cycle FIPP e interferência mínima para criação do caminho de backup. Embora o roteamento de caminhos múltiplos tenha sido usado para reduzir a fragmentação do espectro no EON-SDM, nenhum algoritmo de caminhos múltiplos foi proposto para a proteção do EON-SDM usando o $p$-cycle FIPP e interferência mínima.

Este artigo está organizado da seguinte forma. A Seção 2 revisa os trabalhos relacionados. A Seção 3 introduz o algoritmo MINION. A Seção 4 avalia o desempenho do algoritmo proposto e a Seção 5 conclui o artigo.

\section{Trabalhos Relacionados}

$\mathrm{Na}$ literatura, existem diversos trabalhos que tratam de roteamento multicaminho para redes ópticas, no entanto, poucos trabalhos estudam a proteção destes caminhos.

Em [Gao et al. 2016], é apresentada uma formulação programação linear inteira (ILP) e uma heurística para roteamento multicaminho e alocação de espectro para redes ópticas elásticas protegidas. O esquema proposto atinge uma melhora na utilização de recursos em comparação com os esquemas de proteção tradicionais. Além de multicaminho, os algoritmos utilizaram diferentes formatos de modulação. Os autores em [Goścień et al. 2016], estudaram a sobrevivência de redes ópticas elásticas através de roteamento multicaminho, permitindo a divisão de uma demanda em vários caminhos de roteamento, garantindo a realização de um volume de demanda específico no caso de uma falha de um único enlace. O artigo propõe uma ILP e uma heurística para resolver o problema de sobrevivência em redes ópticas elásticas utilizando multicaminho.

Foram investigados em [Dharmaweera et al. 2016] os ganhos potenciais, empregando conjuntamente com agregação de tráfego e multicaminho para redes ópticas elásticas. Através de simulações, os autores demonstraram que a agregação de tráfego e roteamento multicaminho em conjunto, aumentam a eficiência espectral e reduzem o consumo de recursos em relação aos esquemas existentes. Seis novos algoritmos para resolver o problema de fragmentação e melhorar a probabilidade de bloqueio em redes 
ópticas elásticas foram introduzidos em [Yousefi et al. 2019]. Os algoritmos propostos reduziram a probabilidade de bloqueio (BP) controlando a fragmentação em cada caminho. Os algoritmos dividem uma demanda em várias rotas. Ao oferecer essa oportunidade na alocação e designação de espectro, a probabilidade de bloqueio de conexão é reduzida.

Um esquema de provisionamento de caminhos múltiplos tolerante à falhas para redes ópticas elástica é proposto em [Takeda et al. 2019]. Os algoritmos propostos minimizam os recursos de espectro, permitindo alocar os diferentes números de slots de espectro e diferentes quantidades de capacidade de transmissão para cada caminho. Um algoritmo de proteção para redes ópticas elásticas com multiplexação por divisão espacial usando roteamento híbrido e $p$-cycle foi proposto em [Oliveira and da Fonseca 2019a]. $\mathrm{O}$ algoritmo proposto prioriza o uso de roteamento de caminho único e usa caminhos múltiplos quando nenhum caminho único pode ser encontrado para acomodar a largura de banda solicitada. O algoritmo proposto é comparado a outros quatro algoritmos que usam roteamento de caminho único.

Apesar de alguns trabalhos já terem utilizado multicaminho para prover proteção em redes ópticas elásticas, apenas em [Oliveira and da Fonseca 2019a] foi estudado proteção em redes ópticas elásticas com multiplexação espacial com roteamento multicaminho.

\section{Algoritmo MINION}

O algoritmo introduzido nesta seção é chamado Minimum InterfereNce and hybrId routing for MulticOre Networks (MINION). O algoritmo MINION aceita apenas requisições que possam ser protegidas. Diferentemente do algoritmo proposto em [Oliveira and da Fonseca 2019a] que utiliza $p$-cycles FIPP para prover proteção aos caminhos primários, o algoritmo MINION adicionalmente utiliza interferência mínima na criação do $p$-cycle, priorizando $p$-cycles que usam enlaces disjuntos do caminho protegido ( $p$-cycles transzonais). $\mathrm{O}$ algoritmo prioriza, também, estabelecer conexões através de caminhos simples. No entanto, quando estes não são possíveis, o estabelecimento de conexões pode ocorrer através de roteamento multicaminho.

$\mathrm{O}$ algoritmo MINION modela a disponibilidade do espectro na rede como um multigrafo (Figura 1(a)). O multigrafo é utilizado para manter baixa a complexidade do procedimento de alocação de núcleo e espectro, uma vez que o espectro de todos os núcleos tem de ser levado em consideração durante a atribuição. No multigrafo, um rótulo em uma aresta representa a disponibilidade de um slot.

Na Figura 1(b), o multigrafo é dividido em $C$ multigrafos, onde $C$ é o número de núcleos. Cada aresta nos multigrafos criados representa um slot em um determinado núcleo. Cada um desses multigrafos da Figura 1(b) é transformado em um multigrafo com $N-b+1$ arestas (Figuras 1(c) e 1(d)), onde $b$ é a demanda de largura de banda da requisição em slot e $N$ o número de slots em cada núcleo. Cada aresta do multigrafo da Figura 1(d) representa $b$ arestas contínuas do multigrafo da Figura 1(c). Essa representação garante a contiguidade do espectro na solução. Por fim, cada aresta de cada multigrafo resultante formará um grafo (Figura 1(e)). Os grafos gerados serão utilizados para encontrar os caminhos. Em outras palavras, o multigrafo original é transformado em $C \times(N-b+1)$ grafos, onde $C$ é o número de núcleos entre dois nós.

Cada aresta nos grafos (1(e)) representa uma combinação de $b$ slots em um único núcleo. Esta representação assegura a contiguidade do espectro à solução, um vez que 


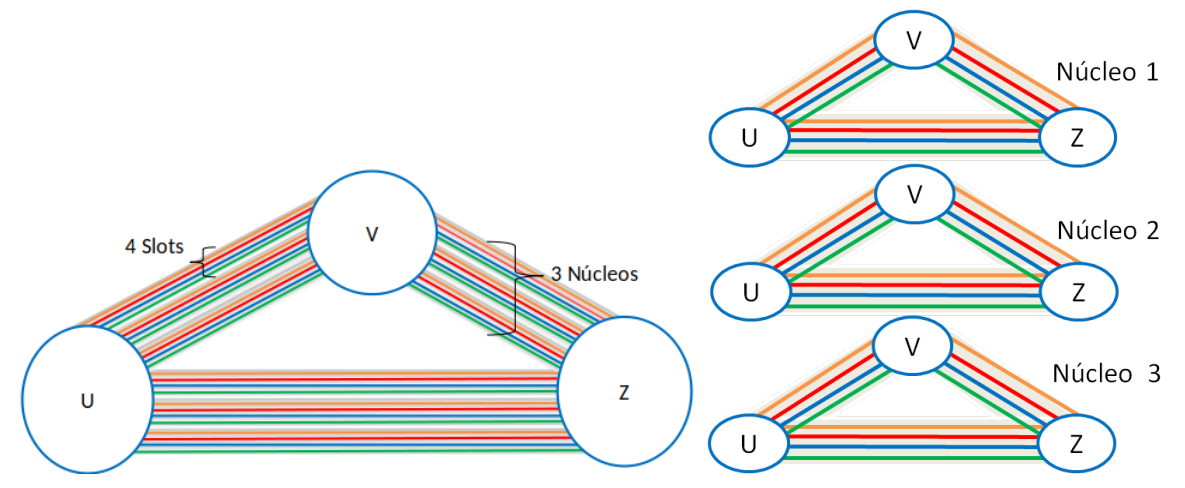

(a) Rede com 3 núcleos e 4 slots.

(b) Os multigrafos, separados por núcleos, cada um representando 4 slots
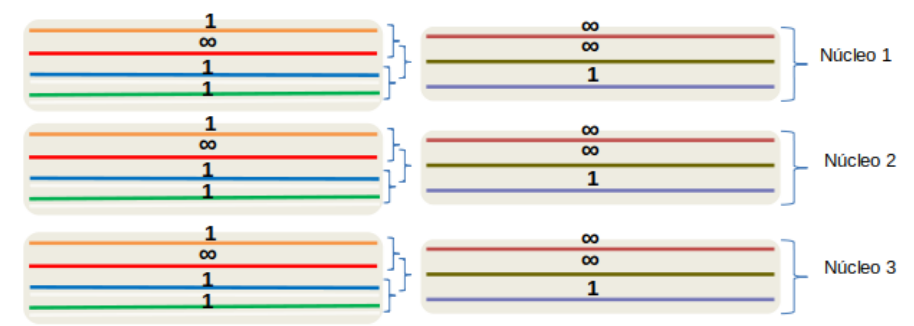

(c) Conjunto de arestas sendo mapeadas em uma aresta
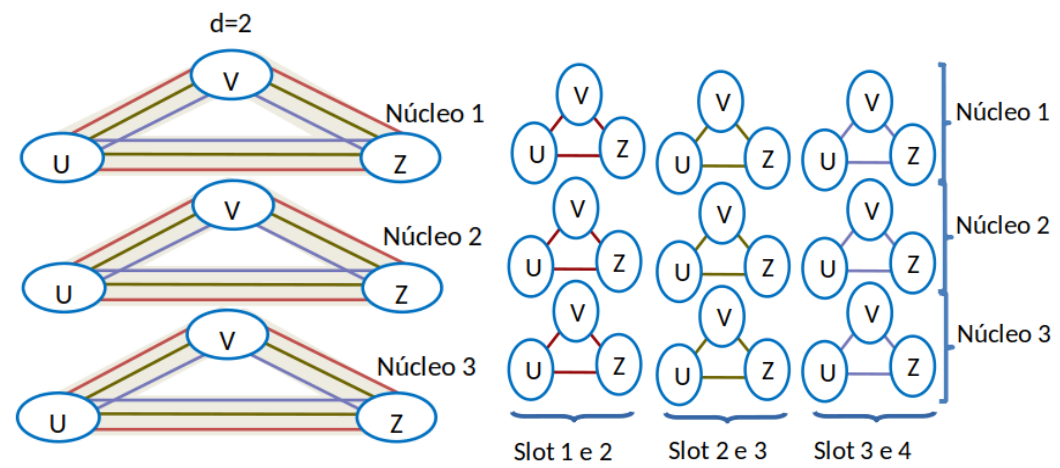

(d) O multigrafo em que o conjunto de arestas são mapeadas em

(e) Grafos gerados uma aresta, restrição de contiguidade.

Figura 1. Transformando multigrafo em grafos.

os grafos gerados são formados por apenas arestas contíguas. Nestes grafos, um valor $\infty$ significa que pelo menos um slot já está alocado enquanto que um valor menor significa que todos os slots estão disponíveis para alocação. O valor $\infty$ pode significar também que o slot não pode ser utilizado, visto que a utilização do mesmo gerará crosstalk inaceitável para a transmissão atual ou para uma transmissão que utiliza um slot no núcleo vizinho. Em outras palavras, um slot não estará disponível se já estiver alocado ou se o valor de crosstalk no slot for inaceitável para uma transmissão bem-sucedida, ou ainda se a utilização deste slot tornar uma transmissão que utiliza um slot do núcleo adjacente inaceitável. 

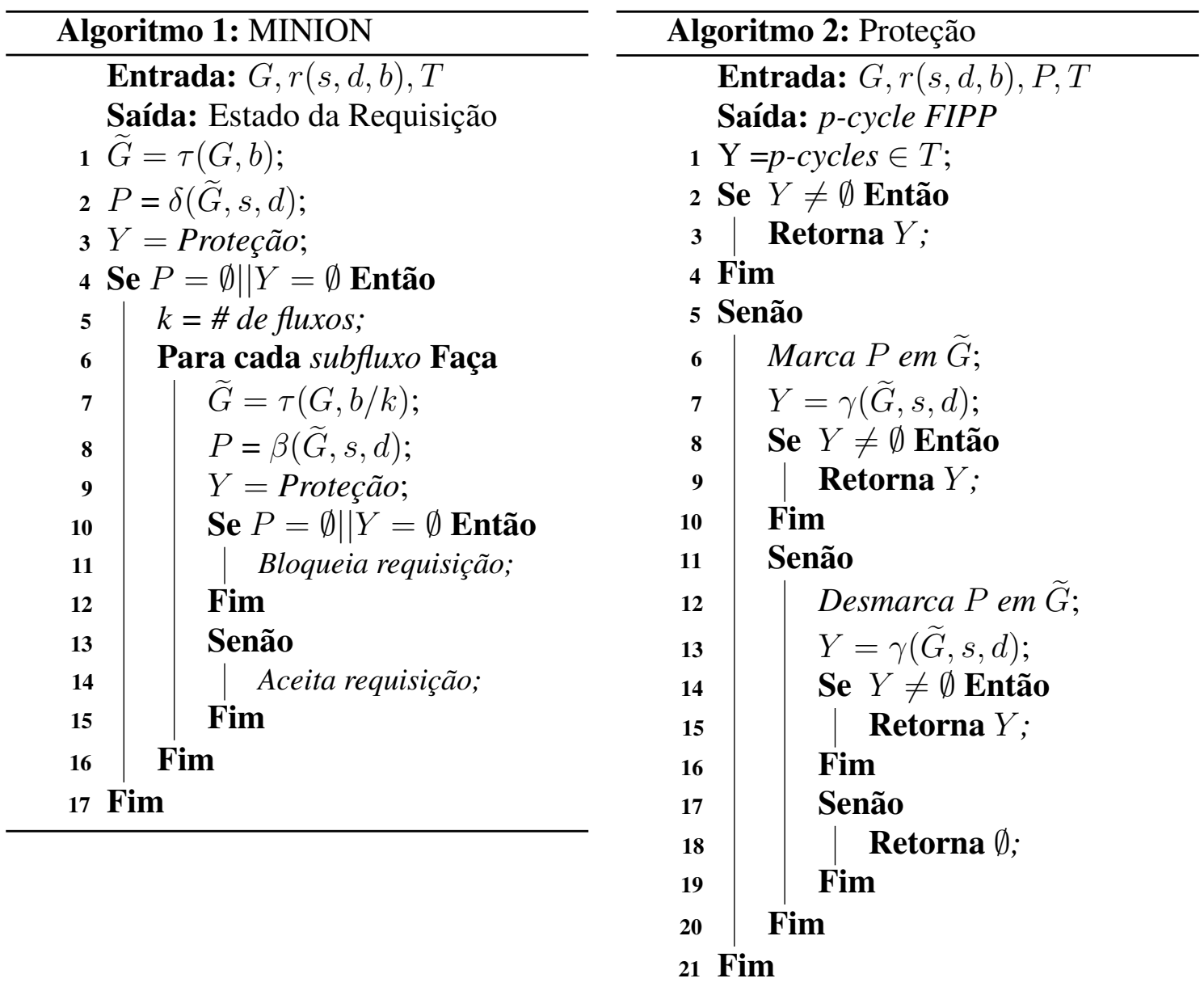

Nos algoritmos propostos, denotamos $G$ como o multigrafo que representa a topologia da rede, e $\widetilde{G}$ os grafos gerados a partir do multigrafo utilizando. Uma requisição é representada através da notação $r(s, d, b)$ onde $s$ representa o nó fonte $s, d$ o no destino e $b$ a quantidade de slots demandada. A notação $T$ representa um conjunto de todos os $p$-cycles estabelecido e ativos no momento da requisição. $\mathrm{O} P$ representa um conjunto de caminhos para estabelecer a conexão, em que $P$ pode ser unitário em caso de roteamento simples. O $Y$ representa um conjunto de p-cycles FIPP escolhido proteger o caminho primário, como o conjunto $P$, o $Y$ pode ser unitário em caso de roteamento simples. A função $\tau(G, b)$, como demonstrado na Figura 1 representa transformação do multigrafo para grafos. A função $\delta(\widetilde{G}, s, d)$ representa o menor caminho nos grafos utilizando o algoritmo de Dijkstra. A função $\beta(\widetilde{G}, s, d)$ representa os $K$ menores caminhos nos grafos utilizando uma adaptação do algoritmo de Dijkstra. Por fim, a função $\gamma(\widetilde{G}, s, d)$ representa os dois menores caminhos disjuntos, encontrados através do algoritmo de Suurballe.

O algoritmo MINION é introduzido como o algoritmo 1. Este algoritmo realiza um roteamento hibrido. Primeiramente, tenta realizar um roteamento simples, se não for possível, tenta realizar um roteamento multicaminho. As linhas 1 a 3 representam o roteamento simples, enquanto as linhas 4 a 16 representam o roteamento multicaminho. $\mathrm{Na}$ linha 1, o multigrafo é transformado em um conjunto de grafos garantindo a restrição de contiguidade no processo de escolha do caminho primário (Ver Figura 1). Na linha 2, encontra-se o menor caminho nos grafos gerados utilizando o algoritmo Dijkstra. Na linha 3 utiliza-se o algoritmo Proteção (algoritmo 2) para encontrar um $p$-cycle para proteger o 
caminho primário. Na linha 4 , garante-se que se o roteamento simples não for possível o roteamento multicaminho é realizado. No roteamento multicaminho a requisição é dividida (Linha 5) em duas. Na linha 6 garante-se que todo fluxo contenha um caminho primário e um $p$-cycle que realiza a proteção. Na linha 7 gera-se os novos grafos a partir da nova quantidade de slots requisitadas para cada fluxo. Na linha 8 encontra-se o menor caminho para cada subfluxo e na linha 9 encontra-se o $p$-cycle que protege cada caminho. As linhas 11 e 12 são as responsáveis por bloquear ou rejeitar a requisição, respectivamente.

O algoritmo proteção (algoritmo 2) é utilizado para encontrar o p-cycle que protegerá o caminho primário. Este algoritmo possui três fases: 1) compartilhamento de um $p$-cycle que ja esta sendo utilizado; 2) criação de um p-cycle transzonal ao caminho a ser protegido; 3 ) criação de um $p$-cycle que usa os mesmos enlaces do caminho primário. $\mathrm{Na}$ linha 1 busca-se dentre os $p$-cycles ativos $T$ um que possa proteger o caminho primário $P$. Se for possível encontrar; na linha 3 retorna-se o $p$-cycle encontrado. Caso contrário, um novo $p$-cycle é criado. Para não sobrecarregar enlaces específicos da rede e, consequentemente, prejudicar requisições futuras; na linha 6 marcam-se os enlaces nos caminhos primários utilizados e tenta-se encontrar um $p$-cycle (linha 7), totalmente disjunto ao caminho $P$. Se for possível encontrar o $p$-cycle, a linha 9 retorna o $p$-cycle, caso contrário, a linha 12 desmarca os enlaces. Na linha 13, busca-se um p-cycle sem considerar a interferência gerada. A linha 15 retorna o $p$-cycle encontrado ou vazio caso o $p$-cycle não seja encontrado.

A complexidade do algoritmo MINION é analisada como a complexidade de procurar caminhos simples mais a complexidade de procurar multicaminhos. A complexidade de procurar caminhos simples (caminho primário e $p$-cycle) é $O(E+V \log V)$. A complexidade de procurar multicaminhos é analisada como: a complexidade de transformar o multigrafo em grafos é $O(E+V)$. Para encontrar os caminhos primários, o algoritmo de Dijkstra é executado em $C \times(N-b)$ grafos, em que a complexidade amortizada de Dijkstra é $O(E+V \log V)$. Da mesma forma, para os $p$-cycles, o algoritmo de Suurballe é executado em $C \times(N-b)$ grafos. Logo, a complexidade de procurar multicaminhos é $(E+V)+2 \times(C \times(N-b) \times(E+V \log V))+E+V$. Como $N, C$ e $b$ podem ser expressados como constantes, a complexidade de procurar multicaminhos é $O(E+V \log V)$ e a complexidade do algoritmo MINION é então $O((E+V \log V)+(E+V \log V))=$ $O(E+V \log V)$.

\section{Avaliação de Desempenho}

Para avaliar o desempenho do Algoritmo MINION, experimentos de simulação foram realizados utilizando o simulador FlexGridSim [Moura and Drummond ]. Em cada simulação 100.000 requisições foram geradas e utilizou-se o método de replicação independente. Adotou-se nível de confiança de $95 \%$ para os intervalos de confiança. As topologias USA (Figura 2(a)) com 24 nós e 43 enlaces bidirecionais foram empregadas e a topologia NSF (Figura 2(b)) com 14 nós e 18 enlaces bidirecionais, as figuras mostram as respectivas distancias entre os nós. Variou-se a carga entre 25 e 400 erlangs em intervalos de 25 erlangs. Os nós de origem e destino para cada requisição de conexão são uniformemente selecionados aleatoriamente a partir dos nós da rede. Assumimos sete tipos diferentes de demandas com taxa de 25/50/125/200/500/750/1000 Gbps. Os enlaces foram compostos por fibras com 7 núcleos e cada núcleo foi dividido em 320 slots. As métricas utilizadas na comparação são relação de bloqueio de banda, relação de crosstalk 
por slot, índice de fragmentação e eficiência energética .

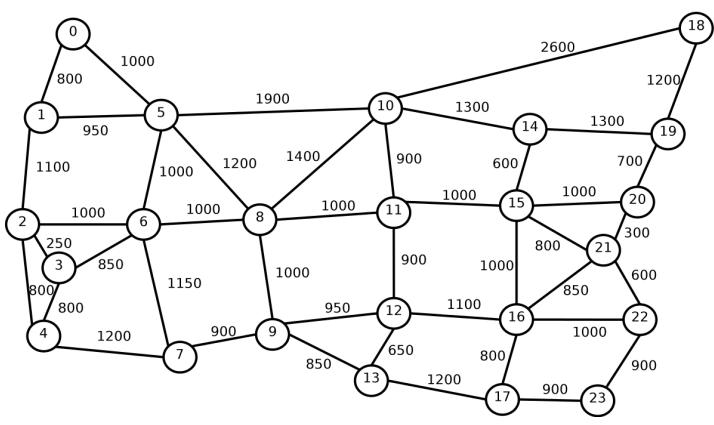

(a) Topologia USA

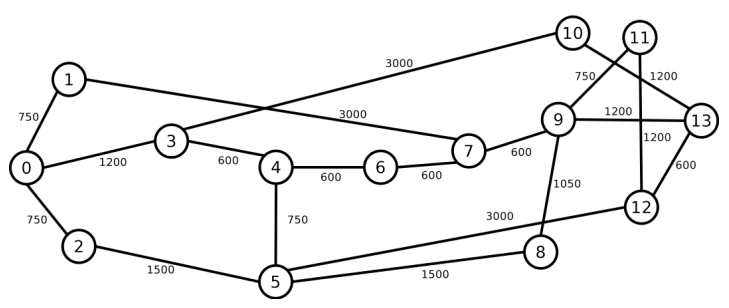

(b) Topologia NSF

Figura 2. Topologias

Nas figuras, as curvas rotuladas como FIPPMC mostram os resultados para as redes que utilizam o algoritmo FIPPMC proposto em [Oliveira and da Fonseca 2019b], as curvas rotuladas como SSCA encontra o caminho primário através do algoritmo proposto em [Tode and Hirota 2014], utilizando $\mathrm{k}=3$ e o caminho de proteção adicionando a capacidade de compartilhamento ao mesmo algoritmo, as curvas rotuladas como Cap-DPP mostram os resultados para as redes que utilizam o algoritmo proposto em [Tan et al. 2016]. As curvas rotuladas como PRIME mostram os resultados para as redes que utilizam o algoritmo PRIME proposto em [Oliveira and da Fonseca 2019a], enquanto as curvas rotuladas como MINION mostram os resultados para as redes que utilizam o algoritmo proposto neste artigo. Salienta-se que os algoritmos FIPPMC e PRIME, utilizam a técnica $p$-cycle FIPP para prover proteção e os algoritmos Cap-DPP e SSCA utilizam caminhos dedicados e caminhos compartilhados, respectivamente. Dos algoritmos comparados apenas o algoritmo PRIME utiliza roteamento multicaminho, pois este foi o único encontrado na literatura para EON-SDM.

A relação de bloqueio da largura de banda (BBR), o crosstalk por slot (CpS), a relação de fragmentação e a eficiência energética foram avaliadas nas simulações. A relação de bloqueio de largura de banda é a porcentagem do tráfego bloqueado em relação à largura de banda total solicitada durante cada simulação. O crosstalk por slot é a proporção média entre os slots afetados pela crosstalk e o número total de slots usados em um link. A relação de fragmentação refere-se ao estado no qual existem slots disponíveis, que não podem ser reunidos para uso para aceitar novas solicitações. A relação de fragmentação é definida como a taxa média entre o número de tipos de demanda que não podem ser aceitos e o número total de tipos de demanda. Eficiência energética é a razão entre a demanda total de tráfego atendida com sucesso na rede e o consumo total de energia da rede.

A Figura 3 mostra a probabilidade de bloqueio para as topologias USA e NSF. Para topologia USA (Figura 3(a)), os algoritmos CaP-DPP e SSCA iniciaram o bloqueio de requisições sob carga de 50 erlangs. O algoritmo FIPPMC, que utilizam $p$-cycle FIPP e roteamento simples, iniciou o bloqueio de requisições sob carga de 125 erlangs. Os algoritmos PRIME e MINION, que utilizam roteamento híbrido iniciaram o bloqueio de requisições apenas sob carga de 150 e 200 erlangs, respectivamente. Sob carga de 200 erlangs o algoritmo MINION produz bloqueio uma ordem de grandeza menor que o algoritmo PRIME, como consequência da utilização de interferencia mínima na criação do 


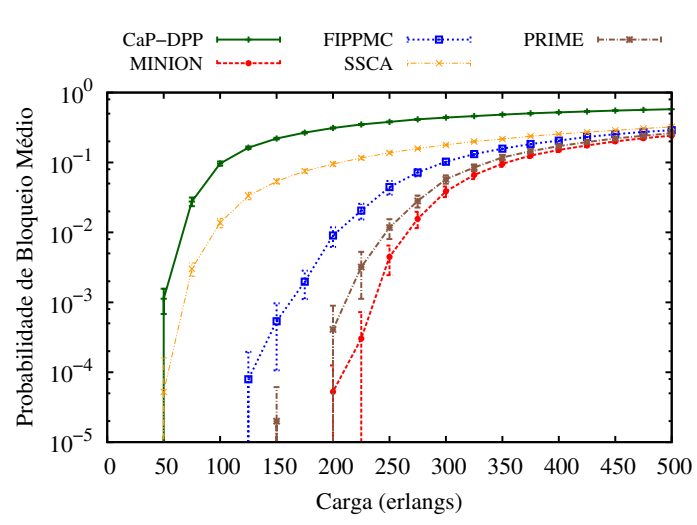

(a) Topologia USA

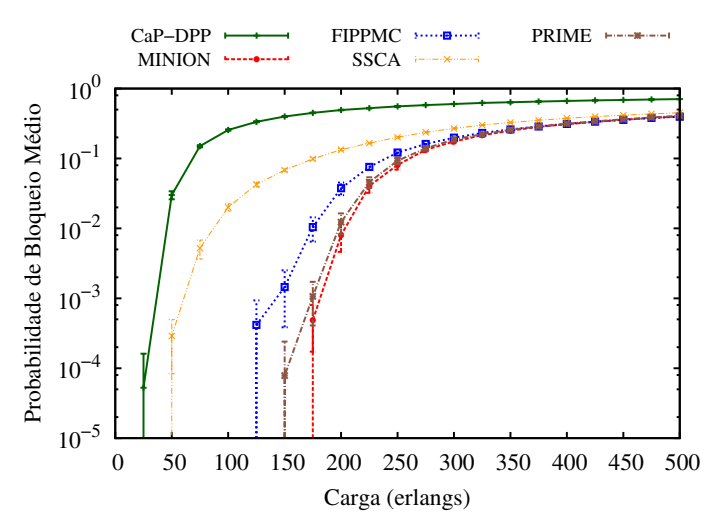

(b) Topologia NSF

Figura 3. Probabilidade de bloqueio

p-cycle FIPP. Os algoritmos que utilizam apenas roteamento monocaminho produzem valores de BBR maiores que os algoritmos que utilizam roteamento híbrido. O algoritmo CaP-DPP produz valores de BBR maiores que o os valores de BBR produzidos pelo algoritmo SSCA, como consequência do compartilhamento de recursos de backup do algoritmo SSCA. O algoritmo MINION produz os menores valores de BBR, evidenciando a influência da utilização conjunta de $p$-cycles, roteamento multicaminho e interferência mínima na criação de caminhos de backup. O algoritmo MINION produz alto compartilhamento dos caminhos de backup, visto que ele produz caminhos de backup maiores. Sob cargas maiores que 350 erlangs os algoritmos PRIME e MINION produzem valores de BBR similares, isto ocorre pois quanto maior a carga maior o compartilhamento de p-cycle.

Para topologia NSF (Figura 3(b)), os algoritmos CaP-DPP e SSCA iniciaram o bloqueio de requisições sob cargas de 25 e 50 erlangs, respectivamente. O algoritmo FIPPMC iniciou o bloqueio de requisições apenas sob carga de 125 erlangs, enquanto que o algoritmo os algoritmos PRIME e MINION iniciaram o bloqueio de requisições apenas sob cargas de 150 e 175 erlangs, respectivamente. Os algoritmos que utilizam roteamento híbrido produzem menores valores de BBR que os algoritmos que utilizam roteamento simples. Sob carga de 150 erlangs, os algoritmo FIPPMC, que utiliza roteamento monocaminho, produz menores valores de BBR que o algoritmo PRIME e MINION, isto ocorre devido a menor conectividade da topologia NSF. Mesmo em uma topologia com baixa conectividade o algoritmo MINION produziu menores valores de BBR que o algoritmo PRIME, evidenciando a vantagem de utilizar interferência mínima e $p$-cycle juntamente com roteamento multicaminho. Como para a topologia NSF, o algoritmo CaP-DPP produz os maiores valores de BBR, como consequência de não utilizar compartilhamento de caminhos de backup e não realizar boas escolhas para encontrar o caminho. O algoritmo SSCA produz o segundo maior número de requisições rejeitadas, influenciado pela não utilização de roteamento multicaminho e pela forma como o algoritmo escolhe os caminhos.

A Figura 4 mostra a relação de crosstalk por slot gerado para as topologias USA e NSF. Para a topologia USA (Figura 4(a)), os valores de CpS gerados pelos algoritmos FIPPMC, PRIME e MINION iniciam com um valor de 0,02 e aumentam até 0,38, 0,41 e 0,47 , respectivamente. O valor de $\mathrm{CpS}$ gerado pelo o algoritmo SSCA inicia com um 


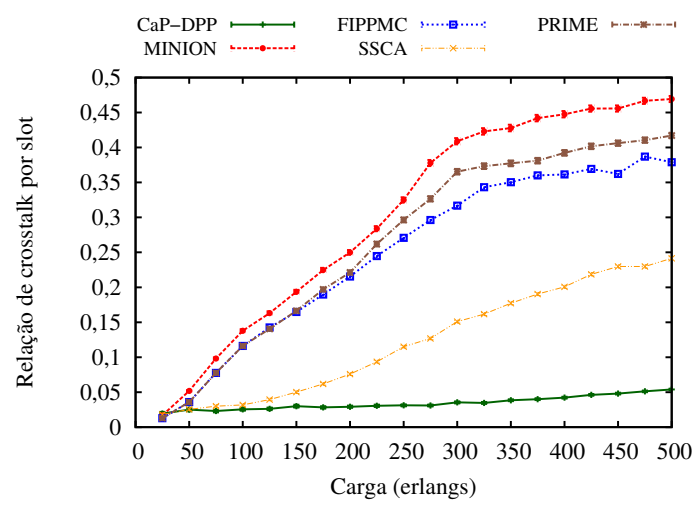

(a) Topologia USA

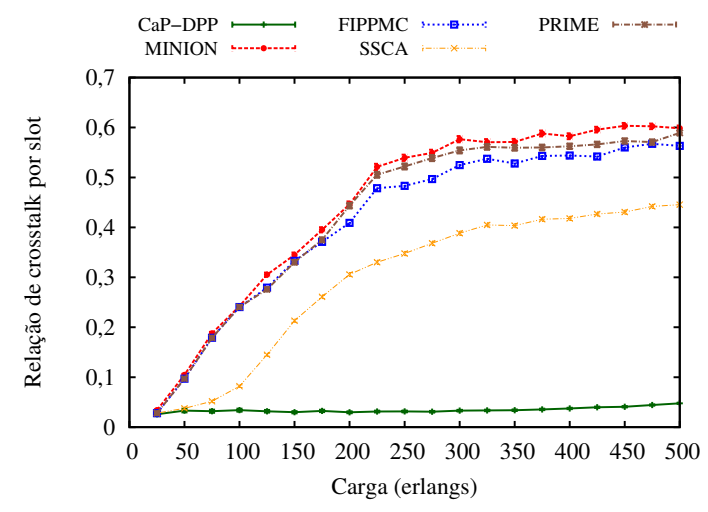

(b) Topologia NSF

Figura 4. Relação de crosstalk por slot

valor de 0,02 e aumentam até 0,24. O algoritmo CaP-DPP produz o menor CPS, influenciado pelo menor número de requisições aceitas. Os algoritmos que utilizam $p$-cycle FIPP produzem os maiores valores de $\mathrm{CpS}$, como consequência da maior utilização de recursos de backup, o que aumenta o número de saltos dos caminhos primários produzidos, e consequentemente aumentam o número de slots que sofrem com crosstalk. Apesar dos algoritmos que utilizam roteamento híbrido produzirem valores de BBR menores, os algoritmos que utilizam roteamento simples produzem valores de $\mathrm{CpS}$ próximos, mostrando que a técnica de proteção tem maior influência no crosstalk do que o tipo de roteamento.

Para topologia NSF (Figura 4(b)), os algoritmos PRIME e MINION iniciam os valores de $\mathrm{CpS}$ em 0,02 e incrementam até 0,6. O algoritmo FIPPMC iniciam os valores de $\mathrm{CpS}$ em 0,02 e incrementam até 0,59. Os algoritmos SSCA e CaP-DPP iniciam os valores de $\mathrm{CpS}$ em 0,02 e aumentam até 0,05 e 0,42, respectivamente. O algoritmo CaP-DPP produz CpS quase constante para todas as cargas simuladas, pois este algoritmo inicia o bloqueio de requisições sob cargas baixas. Como para a topologia NSF, os algoritmos FIPPMC, PRIME e MINION que utilizam p-cycle produzem os maiores valores de $\mathrm{CpS}$, mais uma vez mostrando que com a maior utilização de recursos para caminhos de backup, maior a influência do $\mathrm{CpS}$ para os caminhos primários. No entanto, o crosstalk desses algoritmos ainda estão em um limiar aceitável. Os algoritmos que utilizam roteamento híbrido produzem valores de $\mathrm{CpS}$ próximos ao respectivos algoritmos que utilizam roteamento monocaminho.

A Figura 5 mostra a relação de fragmentação gerada para as topologias USA e NSF. Para a topologia USA (Figura 5(a)), sob altas cargas, o algoritmo PRIME seguido dos algoritmos FIPPMC e MINION, respectivamente, produzem as maiores relações de fragmentação, pois os algoritmos que utilizam $p$-cycle FIPP utilizam mais recursos de backup, apesar dos recursos não serem utilizados, a reserva gera fragmentação. Sob baixas cargas, os algoritmos CaPP-DPP e SSCA produzem a maior razão de fragmentação, isto acontece devido a forma que estes algoritmos encontram os caminhos utilizados. Conforme estes algoritmos diminuem o número de requisições aceitas, o número de desconexões torna-se menor influenciando na fragmentação. Para topologia NSF (Figura 5(b)), sob cargas altas, os algoritmos PRIME, FIPPMC e MINION que utilizam $p$-cycle FIPP produzem as maiores relações de fragmentação, devido ao número de saltos dos caminhos produzidos o que gera um maior estabelecimento de conexões. Sob cargas altas, 


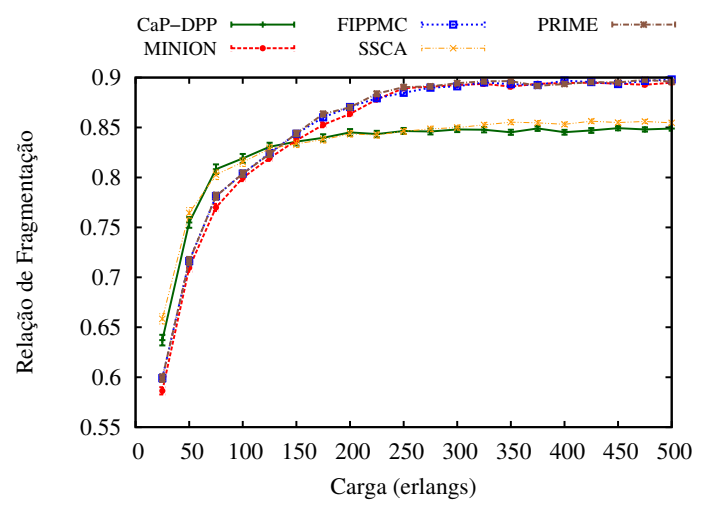

(a) Topologia USA

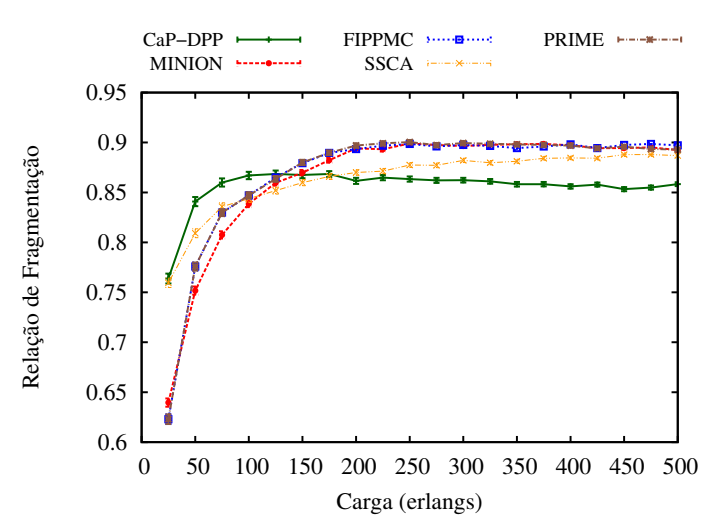

(b) Topologia NSF

Figura 5. Relação de fragmentação

o algoritmo CaP-DPP produz a menor relação de fragmentação, como consequência do maior bloqueio de requisições, implicando em um menor número de estabelecimentos de conexões.

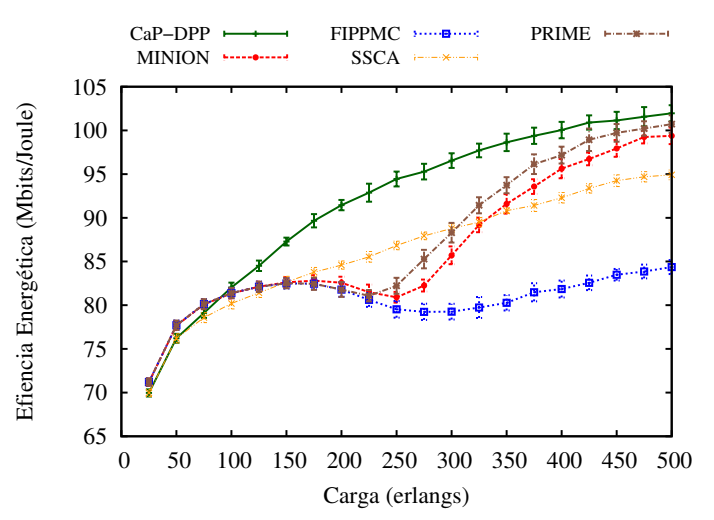

(a) Topologia USA

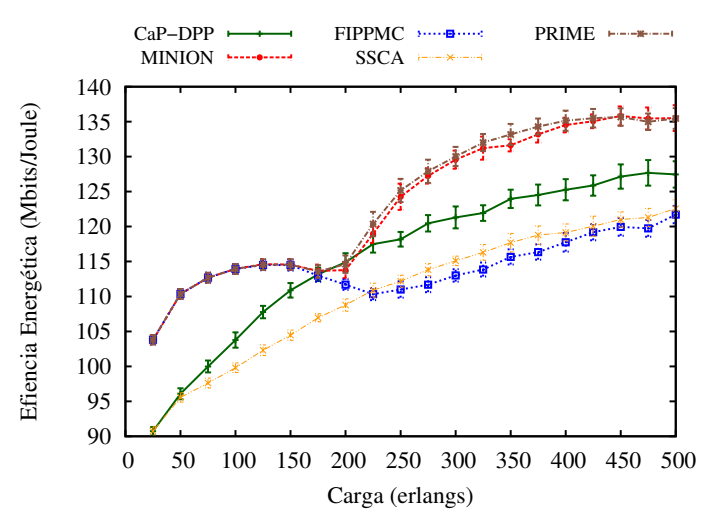

(b) Topologia NSF

Figura 6. Eficiência energética

A Figura 6 mostra a eficiência energética gerada para as topologias USA e NSF. Para topologia USA (Figura 6(a)), sob cargas altas, o algoritmo CaP-DPP produz a maior eficiência energética, isso ocorre devido ao alto número de bloqueio de requisições, que faz com que apenas caminhos menores sejam estabelecidos. Conforme ocorre a necessidade de utilização de roteamento multicaminho pelos algoritmos MINION e PRIME, ocorre uma maior eficiência energética quando comparado ao algoritmo FIPPMC. Entre as cargas 150 e 250 erlangs os algoritmos MINION, PRIME e FIPPMC decrementam a eficiência energética conforme a carga aumenta. Com o aumento do número de requisições bloqueadas, a eficiência energética volta a aumentar. Os algoritmos CAPDPP e SSCA que iniciam o bloqueio de requisições sob baixas cargas produzem eficiência energética sempre em crescimento. Para topologia NSF (Figura 6(b)), sob cargas altas os algoritmos PRIME, FIPPMC e MINION que utilizam $p$-cycle FIPP produzem as maiores razões de fragmentação, devido ao número de saltos dos caminhos produzidos o que gera um maior estabelecimento de conexões. Sob cargas altas, o algoritmo CaP-DPP produz a menor razão de fragmentação, como consequência do maior bloqueio de requisições, 
implicando em um menor número de estabelecimentos de conexões.

\section{Conclusão}

Este artigo introduziu um algoritmo para o estabelecimento de caminhos ópticos em redes ópticas elásticas com multiplexação espacial protegidas por p-cycle FIPP utilizando multicaminho. Enquanto a técnica de $p$-cycle FIPP realiza a restauração rápida de proteção em forma de anel e usa eficientemente a capacidade de proteção em malha como se fosse anel. A técnica multicaminho provê uma eficiente alocação de recursos reduzindo a fragmentação da rede. O algoritmo MINION foi avaliado para diferentes topologias e cargas e seus resultados comprovam que o uso de multicaminhos implica em um maior uso de banda de guarda, já que caminhos diferentes necessitam ser separados por banda de guarda. O maior consumo de recursos na rede, não produz maior bloqueio de requisições, já que a divisão de caminhos utilizando roteamento multicaminho possibilita uma maior facilidade para respeitar as restrições de continuidade e contiguidade de núcleo e espectro em redes ópticas elásticas com multiplexação espacial. Por outro lado, a utilização de multicaminhos gera uma maior complexidade na escolha e na decodificação dos dados.

\section{Referências}

Alvizu, R., Soto, V., Troia, S., and Maier, G. (2018). Enabling multipath optical routing with hybrid differential delay compensation. In 2018 20th International Conference on Transparent Optical Networks (ICTON), pages 1-4. IEEE.

Assis, K., Almeida, R., Waldman, H., Santos, A., Alencar, M., Reed, M., Hammad, A., and Simeonidou, D. (2019). Sla formulation for squeezed protection in elastic optical networks considering the modulation format. IEEE/OSA Journal of Optical Communications and Networking, 11(5):202-212.

Dharmaweera, M. N., Zhao, J., Yan, L., Karlsson, M., and Agrell, E. (2016). Trafficgrooming- and multipath-routing-enabled impairment-aware elastic optical networks. IEEE/OSA Journal of Optical Communications and Networking, 8(2):58-70.

Drummond, A. C., da Fonseca, N., Chen, X., and Jukan, A. (2017). Roteamento multicaminho para provisão eficiente de recursos interdomínio com qualidade de serviço em redes ópticas wdm. In Simpósio Brasileiro de Redes de Computadores e Sistemas Distribuídos (SBRC) 2017, Belém, Pará.

Forecast, C. V. (2019). Cisco visual networking index: Global mobile data traffic forecast update, 2017-2022 white paper.

Gao, T., Huang, S., Guo, B., Li, X., Kong, Q., Zhou, Y., Li, W., and Gu, W. (2016). Survivable multipath provisioning with content connectivity in elastic optical datacenter networks. In 2016 21 st OptoElectronics and Communications Conference (OECC), pages $1-3$.

Gao, T., Zou, W., Li, X., Guo, B., Huang, S., and Mukherjee, B. (2019). Distributed sub-light-tree based multicast provisioning with shared protection in elastic optical datacenter networks. Optical Switching and Networking, 31:39-51.

Goścień, R., Walkowiak, K., and Tornatore, M. (2016). Survivable multipath routing of anycast and unicast traffic in elastic optical networks. IEEE/OSA Journal of Optical Communications and Networking, 8(6):343-355. 
Marom, D. M., Colbourne, P. D., D'errico, A., Fontaine, N. K., Ikuma, Y., Proietti, R., Zong, L., Rivas-Moscoso, J. M., and Tomkos, I. (2017). Survey of photonic switching architectures and technologies in support of spatially and spectrally flexible optical networking [invited]. IEEE/OSA Journal of Optical Communications and Networking, $9(1): 1-26$.

Moura, P. M. and Drummond, A. C. FlexGridSim: Flexible Grid Optical Network Simulator. http://www.lrc.ic.unicamp.br/FlexGridSim/.

Oliveira, H. M. N. S. and da Fonseca, N. L. S. (2019a). P-cycle protected multipath routing, spectrum and core allocation in sdm elastic optical networks. In ICC 2019 2019 IEEE International Conference on Communications (ICC), pages 1-6.

Oliveira, H. M. N. S. and da Fonseca, N. L. S. (2019b). Routing, spectrum and core assignment algorithms for protection of space division multiplexing elastic optical networks. Journal of Network and Computer Applications, 128:78-89.

Pederzolli, F., Siracusa, D., Zanardi, A., Galimberti, G., La Fauci, D., and Martinelli, G. (2019). Path-based fragmentation metric and rsa algorithms for elastic optical networks. IEEE/OSA Journal of Optical Communications and Networking, 11(3):1525 .

Shannon, C. E. (1948). A mathematical theory of communication. Bell system technical journal, 27(3):379-423.

Takeda, K., Sato, T., Shinkuma, R., and Oki, E. (2019). Fault-tolerant multipath provisioning in elastic optical networks. In 2019 24th OptoElectronics and Communications Conference (OECC) and 2019 International Conference on Photonics in Switching and Computing (PSC), pages 1-3. IEEE.

Tan, Y., Zhu, R., Yang, H., Zhao, Y., Zhang, J., Liu, Z., Qu, Q., and Zhou, Z. (2016). Crosstalk-aware provisioning strategy with dedicated path protection for elastic multicore fiber networks. In 2016 15th International Conference on Optical Communications and Networks (ICOCN), pages 1-3.

Tode, H. and Hirota, Y. (2014). Routing, spectrum and core assignment for space division multiplexing elastic optical networks. In Telecommunications Network Strategy and Planning Symposium (Networks), 2014 16th International, pages 1-7.

Xiao, L., Chen, S., Xue, C., Yang, L., and Yang, T. (2019). Comprehensive performance study of elastic optical networks for distributed datacenter with survivability. In Optical Fiber Communication Conference, pages Th2A-23. Optical Society of America.

Yousefi, F., Rahbar, A. G., and Yaghubi-Namaad, M. (2019). Fragmentation-aware algorithms for multipath routing and spectrum assignment in elastic optical networks. Optical Fiber Technology, 53:102019.

Zhan, K., Yang, H., Yu, A., Yao, Q., and Zhang, J. (2019). Multi-path prereserved resource allocation based on tidal traffic prediction in metropolitan optical network. In 17th International Conference on Optical Communications and Networks (ICOCN2018), volume 11048, page 110480U. International Society for Optics and Photonics. 